Review Article

\title{
Effect of Behavioural counselling in Tobacco Cessation
}

\author{
Manjula D.C ${ }^{1}$, Vandana Shekar ${ }^{2}$, Jagat reddy R.C ${ }^{3}$
}

\begin{abstract}
Tobacco cessation is an important component of a comprehensive tobacco control strategy since quitting tobacco is the single most important intervention that can improve the duration and quality of life of every tobacco user. Behavioural counselling is the cost effective way of reducing ill health by providing both immediate and long-term health benefits at the community, national and individual levels. This short communication highlights and provides guidelines to strengthen tobacco cessation activities and various management methods of tobacco cessation in tobacco users.
\end{abstract}

Key words: Behavioural counselling, Tobacco,Quitting.

Tobacco cessation is an important component of a comprehensive tobacco control strategy since quitting tobacco is the single most important intervention that can improve the duration and quality of life of every tobacco user. Various other methods are self-help materials such as handouts, pamphlets, videos, and computer programs, school-based and community-based programs, and the use of the standard " $5 \mathrm{As}$ " and $5 \mathrm{R}$ 's. 5 A's is a brief intervention method of counseling and a very popular approach used to guide the clinician in tobacco cessation counseling.

In Indian scenario and global scenario, cancer patterns in India reveal that the predominance of tobacco related cancers are habit associated so health education in the form of individual counseling is the most essential methods of prevention at the primary level. Behavioural counselling is the cost effective way of reducing ill health by providing both immediate and long-term health benefits at the community, national and individual levels. This short communication highlights and provides guidelines to strengthen tobacco cessation activities and various management methods of tobacco cessation in tobacco users.

Aveyard et al (2012) in a review on individual psychological counselling for cessation of tobacco stated that this type of counselling includes planned personal activities by a qualified cessation counselor and counselling is of 10 minutes duration. This method is very effective in helping the tobacco user by first asking about tobacco users interest in quitting date, Generally sessions lasts for a episode of 4 weeks following a quit
date.Multiple and lengthier meetings are found to be more successful. ${ }^{1}$ Group or individual psychological support can also help people to quit. ${ }^{2}$ The five major steps in this intervention are: 5 A'S They are as follows Ask-about tobacco use. Advice- to quit Assess-commitment and barrier to change.Assist-users committed to change. Arrange-follow up to monitor progress. ${ }^{3}$

5 R's model has been advocated for tobacco users who were unwilling to quit.

They were Relevance, Risks, Rewards, Roadblocks and Repetition.

Relevance : motivate the tobacco user to signify why stopping is personally significant, to be clearly defined as possible. Motivational information has the maximum impact if it is important to the person's disease status or health concerns, age, gender and other vital features e.g; previous experience of using tobacco and individual obstacles in cessation.

Risks: Inform the users to recognize negative consequences of sustained tobacco usage and highlight those that are very significant to the patient.. emphasise on smoking less- nicotine cigarettes or use of various tobacco forms will not eliminate the risks.

Severe risks: breathlessness, asthma, pregnancy, loss of potency, loss of fertility, increased carbon-monoxide.

Extended Risks: heart attacks and strokes, lung and other cancers (larynx, oral cavity, pharynx, oesophagus,), 
chronic bronchitis and emphysema),long-term disability and need for comprehensive care.

Environmental risks: greater risks of lung cancer and heart diseases in spouses; higher risks of smoking by children of tobacco users: increased rate for low birth weight, SIDS, asthma, ear disease and respiratory infections in children of smokers.

Rewards: The Therapist should ask the tobacco users to categorize the probable benefits of stopping tobacco use and converse specific benefits of quitting like improved health, good sense of smell, saving their cash, feel better about yourself. home, car,garments, breath will smell better as a replica for children have healthier babies feel better physically, perform higher in physical manners, decreased aging of skin.

Roadblocks: The Physician should tell the users to find out the barriers to quitting and note specific obstacles to quitting and note essentials of therapy that could deal with problems associated with it.Distinctive barriers are withdrawal symptoms, fear of disappointment, weight gain, unsupported, dejection, on using tobacco.

Repetition: This motivational therapy should be repetitive constantly when an uninterested patient reports the clinic. Tobacco users who were unsuccessful in their past quit attempts should be told that most of them make repeated quit attempts before they are successful ${ }^{4}$

In a systematic (transtheoretical) model developed in 1982 by Prochaska et al., individuals using tobacco products are evaluated in 5 stages with regard to their views and behaviour towards quitting smoking based on nicotine dependence.

a. People who do not plan to quit smoking (pre-contemplation phase)

b. People who have begun to think about giving up smoking (contemplation phase).

c. People who are ready or in the preparation phase to quit smoking (preparation phase).

d. Quitting, trying to quit (action).

e. Maintaining not smoking (maintenance) $)^{5}$
The most common intervention physicians make is to advise cessation (because it will prevent ill health), but a systematic review of randomised controlled trials show that offering support for smoking cessation (such as medication or behavioural support) enhances the rate at which people attempt to stop smoking ${ }^{6}$ The prime aim of interventions to promote cessation at the individual level is to improve health and reduce the ill-health consequences of smoking. ${ }^{7}$ Achieving lifelong tobacco abstinence is an important public health goal. Most studies use 1-yearfollow-ups, but little is known about how good these are as proxies for long-term and life-long abstinence. Also,intervention intensity is an important issue for development of efficient and cost-effective cessation treatment protocols. ${ }^{8}$

\section{Conclusion}

Tobacco cessation facilitates health benefits like improved mental and physical health. Behavioural counselling of two to three minutes can help the quitters to quit than other pharmacotherapy methods like Nicotine replacement therapy where adverse effects are more. However, evidence suggests that a combination of psychotherapy and medication increase the success of quitting success than either of them taken alone.

\section{References}

1. Aveyard P, Raw M. Improving smoking cessation approaches at the individual level. Tob Control.2012; 21(2):252-7.

2. Lancaster $\mathrm{T}$, Lf $\mathrm{S}$. Individual behavioural counselling for smoking cessation ( Review ) Summary of findings for the main comparison.2018;(3):2017-19.

3. Fiore MC, McCarthy DE, Jackson TC, Zehner ME, Jorenby DE,,Mielke M,Smith SS, Giuliani TA,Baker TB, et al. Integrating smoking cessation treatment into primary care : an effectiveness study.Prev Med 2004;38(4):412- 20.

4. Clinical Practice Guideline Treating Tobacco Use and Dependence Update Panel, Liaisons, and Staff. A clinical practice guideline for treating tobacco use and dependence: 2008 update. A U.S. Public Health Service report. Am J Prev Med 2008;35:158-76.

5. Prochaska JO, DiClemente CC, Velicer WF, Rossi JS. Standardized, individualized, interactive, and personalized selfhelp programs for smoking cessation.Health Psychol.1993; 12: 399-405.

6. Aveyard P, Begh R, Farley A, West R.. Brief opportunistic smoking cessation interventions: a systematic review and meta-analysis to compare advice to quit and offer of assistance. Brief opportunistic smoking cessation interventions : a systematic review and metaanalysis to compare.Addiction 2012;107(6):1066-73 
7. Wannamethee SG, Shaper AG, Perry IJ, et al. Smoking as a modifiable risk factor for type 2 diabetes in middle-aged men. Diabetes Care 2001;24:1590-95.

\section{Address of Correspondence}

Manjula D.C ,

Post graduate,

Oral medicine and Radiology,

Indira Gandhi Institute of Dental Sciences,

Email id: manjulasiva1@gmail.com

Phone no: +91 9791057645
8. Nohlert E, Öhrvik J, Tegelberg Å, Tillgren P, Helgason ÁR. Longterm follow-up of a high- and a low-intensity smoking cessation intervention in a dental setting- a randomized trial. BMC Public Health 2013;13(1):592.

\section{Authors \\ 1,5,6Post Graduate Student, Oral Medicine and Radiology, Indira Gandhi Institute of Dental Sciences. \\ ${ }^{2}$ Reader, Oral Medicine and Radiology, Indira Gandhi Institute of Dental Sciences. \\ ${ }^{3}$ Professor and Head, Oral Medicine and Radiology, Indira Gandhi Institute of Dental Sciences.}

How to cite this article : Manjula D.C, Vandana Shekar, Jagat reddy R.C. Effect of Behavioural counselling in Tobacco Cessation. Journal of Scientific Dentistry 2018;8(2):58-60

Source of support : Nil, Conflicts of Interest : None declared 